\title{
NUEVAS DINÁMICAS DE DESIGUALDADY EXCLUSIÓN SOCIOLABORAL DE LAS PERSONAS CON DISCAPACIDAD DURANTE LA CRISIS ECONÓMICA EN ESPAÑA
}

\author{
NEW DYNAMICS OF INEQUALITY AND LABOR EXCLUSION OF PERSONS WITH \\ DISABILITIES DURING THE ECONOMIC CRISIS IN SPAIN
}

\section{Eduardo Díaz Velázquez ${ }^{1}$}

\begin{abstract}
Resumen:
La crisis económica y las sucesivas reformas laborales están implicando cambios sustantivos en el mercado de trabajo en España. Este artículo pretende analizar el impacto que están teniendo dichas transformaciones socioeconómicas y del mercado de trabajo en el acceso al empleo del colectivo de personas con discapacidad, desde el prisma de la teoría sociopolítica de la ciudadanía, evaluando el papel que tienen las políticas de empleo dirigidas a este colectivo para minimizar los efectos de la crisis en un contexto de recorte del gasto público y debilitamiento de los derechos sociales.

Para ello mediante el análisis de datos estructurales y coyunturales del mercado de trabajo en España analizaremos cómo están afectando la crisis económica y las nuevas tendencias del mercado laboral en la estructura ocupacional del colectivo así como la influencia de las políticas de empleo dirigidas al colectivo para amortiguar el impacto de la crisis en su inserción laboral. Ese análisis cuantitativo se complementa con un estudio cualitativo mediante historias de vida de una muestra de personas con discapacidad en edad de trabajar, que nos permite profundizar en los mecanismos articuladores de la discriminación del colectivo y la desigualdad en el acceso al empleo.

Los datos muestran que la crisis está afectando muy negativamente tanto en el acceso al empleo de las personas con discapacidad como en las condiciones laborales de aquellos que se encuentran trabajando, agudizando su precariedad, segmentación y sectorización, así como una mayor dualización laboral dentro del colectivo.
\end{abstract}

\section{Palabras clave:}

Discapacidad, ciudadanía, derechos sociales, mercado de trabajo, crisis económica, dualización laboral, desigualdad, políticas activas de empleo.

\begin{abstract}
:
The economic crisis and the successive labor reforms are involving substantial changes in the labor market in Spain. This article analyzes the impact they are having these socio-economic and labor market access to employment of the group of persons with disabilities, from the perspective of the socio-political theory of citizenship changes, assessing the role that employment policies aimed this group to minimize the effects of the crisis in the context of public spending cuts and dwindling social rights.

To do this by analyzing structural and economic data of the labor market in Spain will discuss how they are affecting the economic crisis and the new labor market trends in the occupational structure of the group and the influence of employment policies aimed at collective to cushion the impact of the crisis on the labor market. This quantitative analysis is complemented by a qualitative study life histories of a sample of persons with disabilities of working age, which gives us insight into the mechanisms of articulating the collective discrimination and inequality in access to employment.
\end{abstract}

1 Lic. En Trabajo Social y en Sociología por la Universidad Complutense de Madrid. Director del Centro Español de Documentación sobre Discapacidad (CEDD) del SIIS- Centro de Documentación y Estudios, Prof. Asociado Universidad Complutense de Madrid, Calle Serrano 140, 28006, Madrid (España), Tel. +34 - 9174524 47; ediaz@siis.net 
The data show that the crisis is negatively affecting both access to employment for people with disabilities and the working conditions of those who are working, sharpening its precariousness, segmentation and zoning, as well as higher labor dualization within the collective.

\section{Keywords:}

Disability, citizenship, social, labor market, economic crisis, labor dualization, inequality, employment policies.

\section{INTRODUCCIÓN}

El trabajo ha tenido un papel central en las políticas públicas dirigidas al colectivo de personas con discapacidad en las últimas décadas, así como en la acción de las entidades sociales, tanto a nivel nacional como internacional, al considerarse el acceso al empleo como elemento integrador de la ciudadanía. Así lo expresa, por ejemplo, la Convención Internacional de los Derechos de las Personas con Discapacidad, cuyo artículo 27 está dedicado al empleo, señalando que los Estados Partes han de garantizar el ejercicio del derecho al trabajo de las personas con discapacidad, adoptando las medidas pertinentes. Entre ellas se encuentran la prohibición de la discriminación y la protección de derechos y aseguramiento del ejercicio de los mismos. También medidas relacionadas con la activación y la preparación laboral, así como con el fomento de su empleabilidad, de acuerdo con los principios de la inclusión activa, frente a una idea de inclusión pasiva, inhibidora de la autonomía, propia de paradigmas anteriores. Algunas de estas medidas son: los programas generales de orientación técnica y vocacional, los servicios de colocación y formación profesional y continua, el apoyo en la búsqueda, obtención y mantenimiento del empleo, la promoción del autoempleo o la promoción de la adquisición de experiencia laboral.

Hasta ahora, las políticas públicas, así como las iniciativas privadas lideradas por el tercer sector de acción social, han desarrollado algunos de estos programas y han facilitado un incremento de los niveles de inserción laboral de las personas con discapacidad en España, si bien no siempre los resultados alcanzados han sido óptimos. Sin embargo, los pequeños logros obtenidos se están dinamitando durante el período de crisis económica. Este artículo pretende analizar cómo está afectando la crisis económica a la estructura ocupacional de la población con discapacidad en edad activa, el impacto que están teniendo las nuevas tendencias del mercado laboral (de flexibilización y precariedad) en el colectivo y el papel que juegan las políticas de empleo en este proceso, analizando los determinantes que pueden influir en la mayor inserción laboral del colectivo y en qué medida las conocidas como políticas activas de empleo dirigidas a este colectivo ayudan a ello.

\section{Las políticas de acceso al empleo de las personas con discapacidad en España}

Más allá del artículo 27 de la Convención, ya mencionado, el capítulo VI del Texto Refundido de la Ley General de derechos de las personas con discapacidad y de su inclusión social (1) está dedicado al derecho al trabajo, promulgándose en los artículos 35 y 36 la garantía del derecho al trabajo, la no discriminación directa e indirecta y la igualdad de trato, derivados de los preceptos de la LIONDAU relativos a la no discriminación y las medidas de acción positiva (artículos 7, 8 y 9). Se siguen distinguiendo fundamentalmente dos modalidades de inserción laboral en la empresa ordinaria (desarrollada en la sección segunda del capítulo) y en el empleo protegido ${ }^{2}$.

Esta nueva ley marco también señala la responsabilidad de los servicios públicos de empleo, de manera directa o indirecta, en la orientación, co-

2 El texto refundido añade también como modalidad el empleo autónomo. 
locación y registro de trabajadores con discapacidad (artículo 38).

Con respecto a la inserción laboral en el empleo ordinario (Sección $2^{a}$ ), se desarrollarán medidas para compensar las desventajas ocasionadas por la discapacidad, adaptación del puesto de trabajo y accesibilidad de la empresa (si bien no se señala tanto la lucha contra la discriminación). En el artículo 41 se enuncian además, como apoyo a esta inclusión, los servicios de empleo con apoyo, regulados por el Real Decreto 870/2007, de 2 de julio, por el que se regula el programa de empleo con apoyo como medida de fomento de empleo de personas con discapacidad en el mercado ordinario de trabajo.

El artículo 42 de esta nueva ley recoge el precepto que la LISMI, en su artículo 38.1, establecía de una cuota de reserva del $2 \%$ de trabajadores con discapacidad en aquellas empresas con más de 50 trabajadores, añadiendo la posibilidad de incluir en este cómputo a aquellos subcontratados mediante empresas de trabajo temporal y señalando la exención de la obligación bien por acuerdos de negociación colectiva o por el acogimiento voluntario a medidas alternativas, tal y como se recogía en la Ley 50/98, Disposición Adicional $11^{a}$.

Ya en los años 80 las empresas tenían dificultades para cumplir con esa cuota, debido al desajuste entre las necesidades y demandas productivas de las empresas y los perfiles laborales y cualificaciones de la población con discapacidad en búsqueda de empleo. Ese problema de "empleabilidad" dificultaba el cumplimiento de la norma, pues las empresas alegaban que para determinadas puestos de trabajo o para el desempeño de tareas en determinados procesos de producción no encontraban a trabajadores con discapacidad con la cualificación necesaria. En consecuencia, en la normativa laboral se desarroIlaron las denominadas "medidas alternativas", que sustituían la obligación de cumplimiento de la norma por otras acciones, habitualmente en la modalidad de empleo protegido, y plasmadas en diferentes normas como el Real Decreto 27/2000, de 14 de enero, de Medidas Alternativas (entre las que se encontraban la compra de bienes o servicios a Centros Especiales de Empleo o la donación a entidades que se ocuparan de la generación de empleo para personas con discapacidad), o el Real Decreto 364/2005, de 8 de Abril, por el que se regula el cumplimiento alternativo con carácter excepcional de la cuota de reserva en favor de los trabajadores con discapacidad (que simplificaba los procedimientos para solicitar la declaración de excepcionalidad en el cumplimiento de la Cuota de Reserva y añadían como nueva excepcionalidad criterios de carácter productivo, organizativo, técnico o económico) De esta manera, para las empresas se hacía mucho más sencillo esquivar el cumplimiento de la cuota y adscribirse a las medidas alternativas señaladas (Laloma, 2007) ${ }^{(2)}$.

En definitiva, se han ido flexibilizando las condiciones para que las empresas cumplan con la cuota de reserva del $2 \%$, de tal manera que el empleo protegido, que en un principio estaba pensado como "pasarela" hacia la integración laboral en el mercado ordinario para las personas con discapacidad (sobre todo intelectual) con más dificultades de inserción, ha cobrado mayor protagonismo. Todo ello en beneficio de las empresas, ante la supuesta imposibilidad de cumplir con el artículo 38.1 de la LISMI.

Volviendo a la Ley General, la sección $3^{\mathrm{a}}$ sobre empleo protegido recoge la definición de los Centros Especiales de Empleo, e incluye la posibilidad de compensación económica a los mismos por parte de los poderes públicos (art. 44), los requisitos para crear un CEE (art. 45) y la posibilidad de creación de enclaves laborales (art. 46). Esta fórmula de Empleo Protegido pretendería acercar los CEE a las empresas ordinarias. Están regulados por el Real Decreto 290/2004 por el que se regulan los Enclaves Laborales como medida de fomento del empleo de las personas con discapacidad. El objetivo de esta fórmula es, principalmente, garantizar la actividad que realicen las personas con discapacidad en los Centros Especiales de Empleo realizando servicios u obras subcontratadas para las empresas ordinarias. 
Para finalizar este rápido repaso por las políticas de empleo y discapacidad, señalar otras medidas de acción positiva reguladas por nuestro ordenamiento, como las subvenciones económicas a la contratación de trabajadores con discapacidad y las bonificaciones en la cuota de la seguridad social de los trabajadores con discapacidad, que suponen un importante incentivo a las empresas para la contratación de personas con discapacidad, cuyo impacto real trataremos de ver con algunos datos.

\section{El acceso a la ciudadanía y al empleo por parte de las personas con discapacidad}

El análisis sociológico de la discapacidad se ha vinculado, sobre todo en el contexto anglosajón, a la idea de ciudadanía (Morris, 1998 ${ }^{(3)}$; Morris, 2005(4); Marks, 2001(5)) y, en particular, a la de la ciudadanía de la diferencia (Young, 2000)(6). Desde esta perspectiva, debemos entender la ciudadanía como estatus formal y como condición sustantiva, en tanto que, si bien una persona con discapacidad tiene garantizados normativamente sus derechos como ciudadano por el mero hecho de serlo, suelen darse desigualdades en el plano real entre personas con discapacidad y el resto de la población para las diferentes dimensiones que configuran la ciudadanía, incluyéndose entre ellas el mundo del trabajo (Díaz Velázquez, 2010) ${ }^{(7)}$. Atendiendo a Marshall (1998: 38-41; 74) ${ }^{(8)}$, la ciudadanía tendría un principio igualador que se antepondría al de la clase social producto del capitalismo, que diferencia a la población en función de su relación con los medios de producción. Si la ciudadanía abarca un conjunto de derechos civiles, políticos y sociales, son estos últimos los que actuarían como mecanismo de compensación para atenuar las desigualdades producidas por el sistema económico.

En ese sentido, son especialmente relevantes las políticas diferenciadas para los colectivos denominados vulnerables, como es el caso de las personas con discapacidad. La acción de las po- líticas sociales públicas, cuyo contenido básico en España en relación con el acceso al empleo hemos enunciado en el apartado anterior, se ha orientado a garantizar (no siempre con suficiente éxito) unas condiciones mínimas de existencia tanto a nivel de atención sociosanitaria como de protección económica. Sin embargo, como se han encargado de subrayar teóricos del modelo social o del más actual modelo de la diversidad funcional, muchas de estas políticas se han centrado más en la protección pasiva y la dependencia más que en fomentar la autonomía personal y la actividad (Oliver, 1998: 31$)^{(9)}$, poniendo el acento en las discapacidades y no en las capacidades de las personas. Las políticas activas y/o de activación han ido sustituyendo paulatinamente a las políticas de protección social, fomentando la inclusión de las personas con discapacidad en el ámbito laboral, si bien no siempre en las mejores condiciones posibles, mediante medidas de acción positiva y de fomento de contratación, entre otras.

¿Por qué existe esa persistencia en centrar buena parte de las políticas de discapacidad en intentar incorporar a las personas con discapacidad o a otros colectivos antes inactivos al mercado laboral? Si bien para el sistema económico es fácilmente justificable en pos del aumento de la productividad y, por consiguiente, de la riqueza, en el caso de los colectivos sociales vulnerables o excluidos que han reivindicado esta opción, se ha defendido por el hecho de haber sido el trabajo el elemento central de participación en la vida pública (Alonso, 2000) ${ }^{3}{ }^{(10)}$, por lo que aquellos colectivos o personas que no accedían a un trabajo, no tenían, al menos en términos simbólicos, el mismo estatus real de ciudadanía, sino que accedían a ella en condición de beneficiarios, con consecuencias diferenciadoras y/o excluyentes para aquel colectivo que se encontrara en dichas

\footnotetext{
3 "La centralidad del trabajo en la definición de la ciudadanía se inscribía (...) como un aspecto constitucionalizado nominal y formalmente, y sólo parcialmente garantizado -aunque de manera sustantiva- con políticas sociales de corte universalista" (Alonso, 2000: 168).
} 
circunstancias, constituyéndose en infraclase o en ciudadanos "de segunda categoría". Es por ello que el derecho al trabajo de las personas con discapacidad (como hemos visto en la Convención o en la Ley General recientemente aprobada) se ha convertido en prioritario, preconizándose la incorporación de las personas con discapacidad al mundo laboral en la medida en que tengan posibilidades para producir bienes o servicios de valor (Abberley, 1998: 87) ${ }^{(11)}$.

Sin embargo, en el contexto de crisis actual ha habido una radical fractura del vínculo entre el trabajo y la ciudadanía (universal) que ya se estaba debilitando anteriormente con la aplicación de políticas económicas neoliberales de reducción del gasto social pero también de disminución y regresividad de los ingresos fiscales, lo que ha propiciado que las condiciones laborales de toda la población hayan empeorado significativamente y el acceso al empleo se haya hecho cada vez más restringido. ${ }^{4}$

Las mayores dificultades de acceso al empleo, su acceso en condiciones precarizadas y los recortes en las prestaciones sociales y económicas, han agudizado la desigualdad social y la exclusión social de colectivos vulnerables como el de las personas con discapacidad, pues aunque éste es un fenómeno multidimensional, tiene su eje central en las relaciones de producción y en las de distribución y consumo (Castells, 2001) ${ }^{(12)}$. Tal y como señala Tezanos $(2001)^{(13)}$, estas transformaciones sociales (en particular, las del mercado de trabajo) implicarían una dualización social que separa a los incluidos y a los excluidos. Dualización que no tiene como eje vectorial el acceso o no al empleo, sino el acceso a un empleo de calidad y el no acceso o acceso a un empleo precario. Precisamente, con este trabajo, trataremos

4 El nuevo modelo postfordista, flexible, internacionalista o globalizado, de constante innovación tecnológica y en el que se dan más tasas de beneficio en el ámbito de la economía financiera que en el de la economía real, ha contribuido a generar una crisis del mundo laboral y de la ciudadanía social que ha tenido, también, significativas repercusiones en las personas con discapacidad. de hacer visible cómo esa dualización social y/o laboral está afectando al colectivo de personas con discapacidad.

\section{MetodoloGía}

Para analizar la situación laboral de las personas con discapacidad y las nuevas tendencias que se consolidan en los últimos años en el mercado de trabajo, producto tanto de la crisis económica como de las reformas laborales aprobadas, vamos a hacer un análisis de datos estadísticos, recopilados a través de diversas fuentes oficiales secundarias a nivel nacional y europeo, que se acompañará a su vez de algunos extractos del discurso de algunas entrevistas en profundidad realizadas para el Proyecto Qualitydes, que sirven para comprender la vivencia de las personas con discapacidad en el mercado de trabajo.

En los últimos años en España se han mejorado las fuentes de información estadística sobre discapacidad y, en particular, aquellas relativas a sus condiciones laborales. A la Encuesta sobre Discapacidades, Autonomía personal y situaciones de Dependencia (EDAD-2008), le han sucedido otras operaciones estadísticas de enorme valor instrumental para conocer la situación laboral de las personas con discapacidad en España. Sin embargo, uno de los mayores problemas para analizar la realidad de la población con discapacidad es cómo entendemos y, a la postre, medimos y operativizamos la discapacidad.

El concepto de discapacidad es un constructo social, en evolución, cuya definición puede variar según el contexto histórico, geográfico, cultural y social en el que nos encontremos. En los últimos años se ha consolidado la idea de que la discapacidad es resultado de la interacción entre el estado funcional de la persona y su entorno físico y social, como se ha reflejado, si bien con matices, en documentos internacionales de la ONU o de la OMS, ya sea, respectivamente, en la Convención Internacional sobre los Derechos de las Personas con Discapacidad en su Preámbulo o en la Cla- 
sificación Internacional del Funcionamiento, CIF (OMS, 2001) $)^{(14)}$.

Sin embargo, el problema surge al operativizar esa concepción de la discapacidad: cómo medir (ya sea en una encuesta o en un registro) ese producto de la interacción entre el estado funcional de la persona y su entorno. Nos vamos a encontrar desde fuentes estadísticas que optan por una operativización restringida, en las que se considera que las personas con discapacidad son aquellas que se encuentran reconocidas y registradas como tales, es decir, que cuentan con un certificado de reconocimiento de una discapacidad superior o igual al 33\% de acuerdo con las formas de medición del sistema de valoración oficial (como en la serie 'El empleo de las personas con discapacidad'), hasta algunas que consideran personas con discapacidad a aquellas que tienen alguna limitación para realizar actividades cotidianas o "normales" debido a algún problema de salud o enfermedad que dure o vaya a durar al menos 6 meses, sin necesariamente definir ni clasificar cuáles serían esas actividades cotidianas. Estas diferentes formas de entender la discapacidad van a implicar dificultades comparativas de los datos disponibles, aunque no restan por ello a las fuentes el potencial analítico global que tienen para conocer la situación laboral de las personas con discapacidad, pues se encuentran tendencias COmunes en todas las fuentes: una desigualdad en el acceso al trabajo condicionada por las limitaciones en la actividad por motivos de salud o discapacidad, no tanto porque esas limitaciones afecten a la realización del trabajo, sino por las barreras experimentadas en el entorno.

\section{Diversas fuentes que miden el mismo fenómeno, pero de distinta forma.}

A continuación, presentamos cada una de las fuentes y sus diferentes formas de operativizar la discapacidad:

- La Estadística sobre el Empleo de las Personas con Discapacidad (EPD) ${ }^{(15)}$, que realiza ac- tualmente el Instituto Nacional de Estadística (INE), utiliza la información derivada de una integración de los datos estadísticos proporcionados por la Encuesta de Población Activa con los datos administrativos registrados en la Base de Datos Estatal de Personas con Discapacidad (BEPD), junto a datos sobre medidas que fomentan el empleo y el empleo protegido provenientes de la Tesorería General de la Seguridad Social (TGSS), información sobre percepción de prestaciones procedente del Registro de Prestaciones Sociales Públicas del Instituto Nacional de Seguridad Social (INSS) y datos sobre dependencia del Sistema para la Autonomía y Atención a la Dependencia (SAAD). Esta serie, al recoger los datos administrativos registrados en la Base de Datos Estatal de Personas con Discapacidad (BEPD), analiza a la población en edad activa 'registrada', con certificado de discapacidad, esto es, aquellas que tienen reconocido un grado de discapacidad superior o igual al $33 \%$, según el procedimiento de valoración establecido por RD 1971/1999 modificado por RD 1856/2009.

- La Estadística sobre el Salario de las Personas con Discapacidad (EPD) ${ }^{(16)}$, que realiza actualmente el INE, analiza la ganancia salarial de los trabajadores con discapacidad por cuenta ajena y lo hace en términos comparativos con los trabajadores sin discapacidad. Esta operación utiliza la información derivada de una integración de los datos estadísticos proporcionados por la Encuesta de Estructura Salarial con los datos administrativos registrados en la Base de Datos Estatal de Personas con Discapacidad (BEPD) y con los facilitados por la Tesorería General de la Seguridad Social sobre fomento al empleo. Al igual que la anterior, recoge los datos administrativos registrados en la Base de Datos Estatal de Personas con Discapacidad (BEPD), con lo cual analiza a la población en edad activa 'registrada', con certificado de discapacidad, esto 
es, aquellas que tienen reconocido un grado de discapacidad superior o igual al $33 \%$, según el procedimiento de valoración establecido por RD 1971/1999 modificado por RD $1856 / 2009$.

- La estadística sobre contratación de personas con discapacidad ${ }^{(17)}$ y el Informe Anual del Mercado de Trabajo de las Personas con Discapacidad $^{(18)}$, que son elaborados por el Observatorio de las Ocupaciones del Servicio Público de Empleo Estatal (SEPE), nos permiten observar las nuevas tendencias en el mercado de trabajo. Al tratarse de un registro administrativo de contratación y demanda de empleo, cuantifica la población con discapacidad como aquella registrada como persona con discapacidad en dichos servicios públicos de empleo.

- La Encuesta sobre Integración Social y Salud, EISS-2012 en España ${ }^{19)}$, que pertenece al European Disability and Social Integration Module (EDSIM), módulo específico de la Encuesta Europea de Salud que incluye, en relación con el empleo, información comparable a nivel europeo sobre actividad, ocupación y desempleo, inserción laboral, estructura ocupacional y dificultades de acceso al mercado laboral y a la formación, entre otras dimensiones. La Encuesta sobre Integración Social y Salud, EISS-2012 identifica y caracteriza a las personas con discapacidad, entendiendo este concepto dentro del marco de la CIF, como aquellas que señalan su condición de salud (enfermedad, problema de salud crónico y/o limitación en las actividades básicas) como una limitación para la realización de una serie de actividades de la vida diaria.

- La Encuesta de Condiciones de Vida de la UE (EU-SILC) -Statistics on Income and Living Conditions (EU-SILC) ${ }^{(20)}$ - se puso en funcionamiento en 2004 en sustitución del Panel de
Hogares de la Unión Europea, POGHE y agrupan en la actualidad a todos los miembros de la UE, más Islandia y Noruega. Si bien estas estadísticas surgen con el propósito principal de supervisar los progresos que realizan los Estados miembros de la UE en la lucha contra la pobreza y la exclusión social, entre sus datos se integran variables relacionadas con el empleo, la formación o el estado de salud de la población. La Encuesta de Condiciones de Vida (ECV) es, en el caso español, la fuente de datos de la EU-SILC. Si bien la ECV y el conjunto de las estadísticas EU-SILC no hacen referencia expresa a la cuestión de la discapacidad, la propia Comisión Europea ha defendido la validez de esta encuesta para el análisis de las cuestiones relacionadas con la situación laboral de las personas con discapacidad, en la medida en que su aproximación a la medición de la misma coincide con el modelo social de la discapacidad (Zaidi, 2011) ${ }^{(21)}$. En ese sentido, la Encuesta de Condiciones de Vida consideraría como personas con discapacidad a aquellas que tienen alguna limitación para realizar actividades cotidianas o "normales" debido a algún problema de salud o enfermedad que dure o vaya a durar al menos 6 meses, aunque sin definir las actividades cotidianas o normales, por lo que éstas quedan condicionadas a la interpretación del entrevistado.

- La Estadística Europea de Políticas del Mercado de Trabajo (PMT) -en inglés, The labour market policy (LMP) database ${ }^{(22)}$-, ha sido diseñada por la Oficina Estadística de la Unión Europea (EUROSTAT), para proporcionar información sobre las intervenciones del mercado laboral que se dirigen a los grupos de personas con dificultades en el mercado de trabajo. Contiene datos para todos los países de la UE con respecto a empleo con apoyo y rehabilitación, cuantificando las ayudas, beneficiarios e importes destinados a empleo con apoyo y rehabilitación para todos los países de la UE, 
para personas con discapacidad acreditada según los criterios de cada país.

- Anuario de Estadísticas del Ministerio de Empleo y Seguridad Social ${ }^{(23)}$. Este anuario recopila los principales datos sociolaborales de España sobre mercado de trabajo, inmigración y emigración, formación profesional y medidas de apoyo al empleo; condiciones de trabajo y relaciones laborales y prestaciones de seguridad social y otra protección social y ofrece las Cuentas Integradas de Protección Social en términos SEEPROS. De este anuario se obtienen también los principales datos sobre Medidas de Apoyo a la Creación de Empleo (ACE), que ofrece información, a nivel autonómico, en relación con las actuaciones establecidas por el Ministerio de Empleo y Seguridad Social en materia de apoyo a la creación de empleo, y, dentro de estas, a las diferentes políticas de integración laboral de las personas con discapacidad. De esta fuente se recogen también los datos de las políticas de integración laboral de las personas con discapacidad (a las cuales sólo pueden acceder las personas con certificado de discapacidad).

\section{ResultadOS:}

\section{La situación laboral de las personas con discapacidad}

A continuación pasamos a exponer algunos de los principales datos analizados de las fuentes mencionadas en el apartado anterior.

Menor participación en el mercado de trabajo, aunque ha aumentado en los años de crisis

Todas las fuentes estadísticas disponibles evidencian una significativa diferencia en la participación laboral de las personas con discapacidad con respecto a las personas sin discapacidad. La tasa de actividad del colectivo es del $36,6 \%$ frente al 77,0\% del resto de la población, según datos de 2012. Sin embargo, este indicador ha experimentado una evolución ascendente durante los años de la crisis (2008-2012), incrementándose en más de tres puntos (del 33,4 al 36,6), probablemente porque en el contexto de crisis población económicamente inactiva se incorporó al mercado de trabajo como demandante de empleo. Habrá que ver en el futuro el comportamiento de este indicador, de cara a observar si asistimos a un cambio estructural de la relación de las personas con discapacidad con la actividad económica o si el crecimiento experimentado estos últimos años en la tasa de actividad ha respondido más a una situación coyuntural.

\section{Gráfico 1}

Evolución de la tasa de actividad en función de la discapacidad. 2008-2012

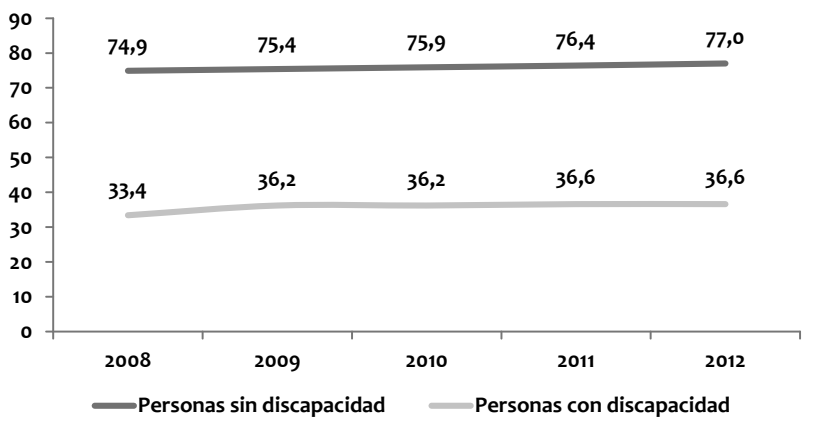

Fuente: El empleo de las personas con discapacidad. Instituto Nacional de Estadística.

Si bien en comparativa con los países de la OCDE la tasa de actividad de las personas con discapacidad en España es significativamente más baja, el comportamiento de este indicador no es homogéneo para todo el país, sino que se observan grandes desigualdades territoriales determinadas en cierta medida por las desigualdades económicas que operan entre comunidades autónomas. Las tasas de actividad más elevadas las encontramos en comunidades como Madrid, País Vasco, Navarra o Baleares (por encima del $40 \%)$. 


\section{Aumento significativo de la tasa de paro durante la crisis}

El aumento de la tasa de actividad no ha conllevado un incremento de la tasa de empleo, sino que ésta se ha visto reducida significativamente al $24,5 \%$ (frente al $57 \%$ de las personas sin discapacidad), reduciéndose entre 2008 y 2012 en 3,5 puntos porcentuales.

Eso ha implicado que la tasa de paro se duplicara (del $16,3 \%$ al $33,1 \%$ ), empujada tanto por la destrucción de empleo como por el crecimiento de la proporción de personas con discapacidad activas. Comparando con los países de nuestro entorno, encontramos que España tiene menos actividad, menos empleo y más paro.

\section{Gráfico 2}

Evolución de la tasa de paro en función de la discapacidad. 2008-2012

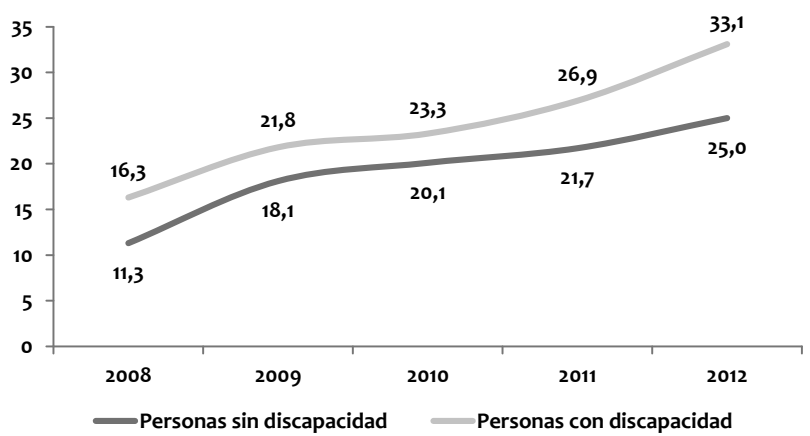

Fuente: INE. El empleo de las personas con discapacidad.

Si atendemos a los datos de parados registrados, demandantes de empleo en los Servicios Públicos de Empleo, podemos constatar también ese incremento. Para el año 2012 había 131.514 personas con discapacidad paradas demandantes de empleo, multiplicándose por tres desde 2005 hasta la actualidad. Lo más destacable es que de ellos, 78.941 (un 60\%) son parados de larga duración (más de un año en paro), incrementándose esa cifra en un 30,3\% entre 2011 y 2012.

\section{Gráfico 3}

Evolución del número de personas con discapacidad paradas demandantes de empleo. 2005-2012

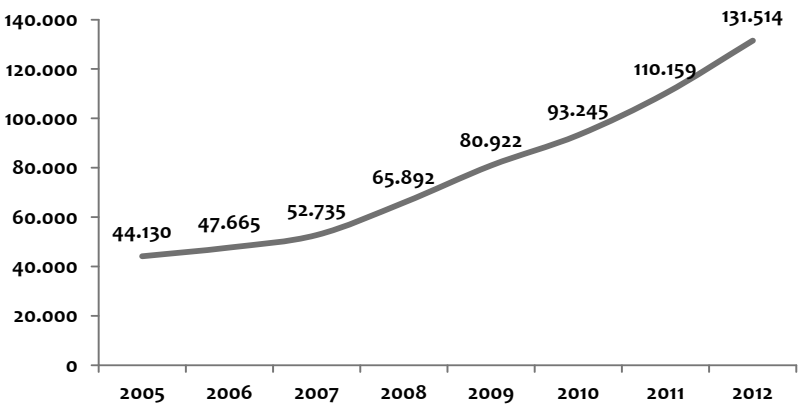

Fuente: INE. El empleo de las personas con discapacidad.

Las nuevas tendencias del mercado laboral se caracterizan por una mayor precariedad e inestabilidad

La crisis económica y las reformas laborales, como decíamos, tienen como consecuencia una progresiva transformación del mercado de trabajo. Las nuevas tendencias en la contratación son: mayor índice de rotación (de 1,94 en 2012, aunque aún así ligeramente más bajo al de la población sin discapacidad, de 2,38). Esto se debe a la mayor eventualidad de la contratación (tanto en empleo ordinario como en empleo protegido). De estar en 2007 la tasa de temporalidad por debajo del $83 \%$, ha pasado casi al $90 \%$ en 2012 .

\section{Gráfico 4}

Evolución de las tasas de estabilidad y temporalidad en la contratación de personas con discapacidad (2007-2012)

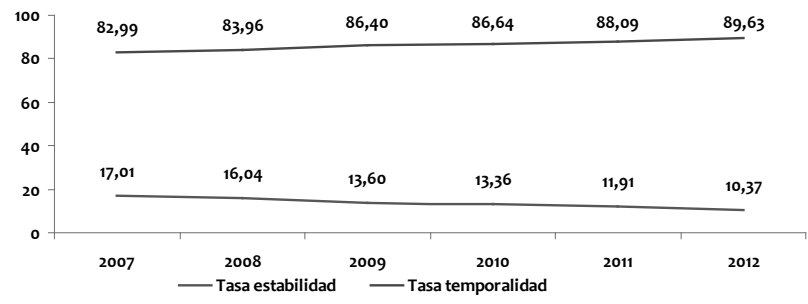

Fuente: Informe Anual del Mercado de Trabajo de las Personas con Discapacidad 2013. Servicio Público de Empleo Estatal (SEPE). 
Este dato contrasta con las cifras de estabilidad y temporalidad de la población que se encuentra trabajando (cuatro de cada cinco tienen contrato indefinido). En estos años ha crecido la proporción de personas contratadas a tiempo indefinido, fundamentalmente por la destrucción de empleos temporales más que por la consolidación de trabajadores fijos.

\section{Gráfico 5}

Evolución de las tasas de estabilidad y temporalidad en la población asalariada con discapacidad (2007-2012)

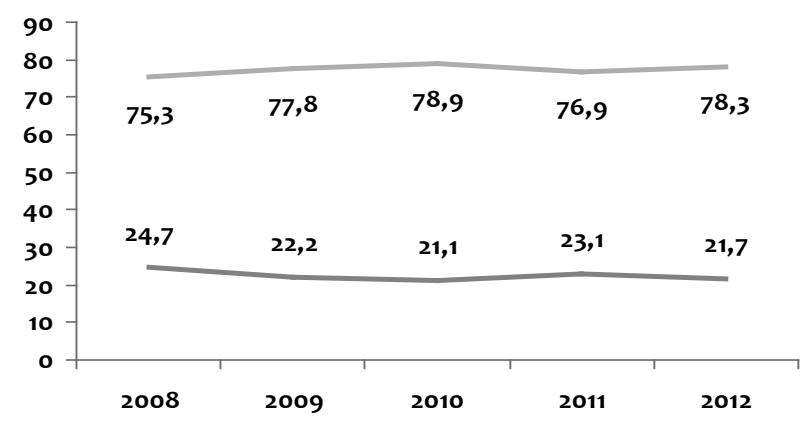

Fuente: El empleo de las personas con discapacidad. Instituto Nacional de Estadística.

Otro cambio fundamental en las nuevas contrataciones ha sido el del incremento de la jornada a tiempo parcial, pasando del $27,9 \%$ en el 2008 al 38,4\% en apenas cuatro años (2012). Este indicador sirve para conocer la precariedad en la contratación, teniendo en cuenta que el tipo de jornada determina el nivel de ingresos y que muchos contratos a tiempo parcial no alcanzan unos ingresos mínimos como para vivir dignamente.
Gráfico 6

Evolución de los nuevos contratos realizados a personas con discapacidad según tipo de jornada (2008-2012)

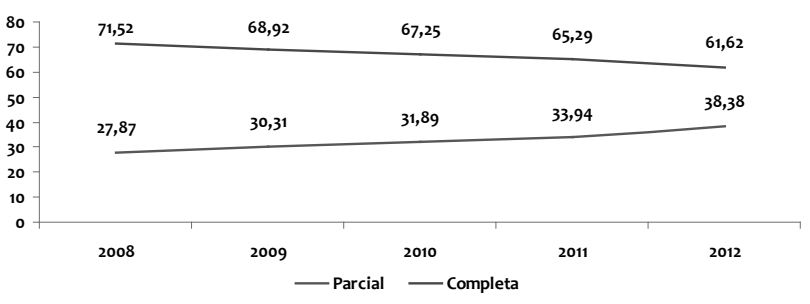

Fuente: Informe Anual del Mercado de Trabajo de las Personas con Discapacidad 2013. Servicio Público de Empleo Estatal (SEPE).

Por el contrario, para el total de la población con discapacidad ocupada, sólo el 17,6\% trabaja a tiempo parcial en 2012, si bien esta cifra ha aumentado en casi cuatro puntos en los últimos años.

\section{Segmentación y sectorización en el mercado laboral}

El tipo de contrato según su duración (mayoritariamente temporal) así como el tipo de jornada (cada vez más frecuente la parcial) nos dan una idea de la escasa calidad del empleo generado para el colectivo de personas con discapacidad. Pero es importante analizar también el tipo de ocupación y de actividad económica en el que se genera empleo para el colectivo.

Si analizamos la contratación realizada a personas con discapacidad por sectores de actividad, podemos observar que ésta se encuentra muy focalizada en actividades económicas del sector servicios (edificios, servicios sociales, transporte, servicios postales o alimentación). La actividad económica que genera un mayor número de contratos para personas con discapacidad es la que engloba los servicios a edificios y actividades de jardinería, con más de 21.000 contrataciones, lo que supone un $4 \%$ del total de contrataciones del sector y cerca del $15 \%$ de todos los contratos realizados a personas con discapacidad. Le siguen en 
orden de importancia las actividades de servicios sociales sin alojamiento, que representan casi el $10 \%$ de todas las contrataciones realizadas a personas con discapacidad.

Desde el punto de vista de la ocupación de las nuevas contrataciones, podemos observar que las mismas tienden a segmentarse en los niveles de ocupación más elementales (que requieren baja cualificación), siendo las más habituales las contrataciones para personal de limpieza de oficinas, hoteles y otros establecimientos similares (más de 23.000 contrataciones, que representan el 15\% del total), seguidas de los peones en industrias manufactureras (más de 11.000 contratos, que suponen el $7 \%$ de todos los contratos a personas con discapacidad).

La ocupación mayoritaria entre las personas con discapacidad es la relacionada con los servicios de restauración, personales, de protección y de ventas, aunque su frecuencia es un 13\% inferior a la de las personas sin discapacidad (19,8 frente a 22,7$)$. Le siguen, en orden de importancia, la relacionada con las ocupaciones elementales (de baja cualificación), en las que desempeña su trabajo el 19,4\% de los ocupados con discapacidad.

Existen diferencias significativas entre personas con y sin discapacidad según los diferentes niveles de cualificación. La presencia de las personas con discapacidad en las ocupaciones de mayor cualificación es muy inferior a la de las personas sin discapacidad. En el caso de los directores y gerentes, la brecha de discapacidad es de 0,55, lo que significa que las personas con discapacidad tienen un $45 \%$ menos de probabilidades de acceder a estos niveles ocupacionales que las personas sin discapacidad (un 2,7 frente a un 4,9). También es inferior la presencia, en términos relativos, de trabajadores con discapacidad como técnicos y profesionales científicos e intelectuales y como profesionales de apoyo, en ambas más de un 20\% por debajo que los ocupados sin discapacidad.

Si ponemos en relación los dos extremos de la pirámide ocupacional, podemos observar una diferencia significativa entre la proporción de per- sonas con y sin discapacidad entre las ocupaciones menos cualificadas (las relacionadas con las ocupaciones elementales) y las más cualificadas (las que corresponden a directores y gerentes). En el caso de las personas con discapacidad, por cada trabajador que ocupa un puesto directivo o de gerencia hay 7,2 personas que ocupan puestos de ocupaciones elementales; en el caso de las personas sin discapacidad, la ratio es de 2,6 trabajadores en ocupaciones elementales por cada directivo.

\section{Gráfico 7}

Evolución de la ratio de trabajadores no cualificados/directivos con y sin discapacidad. 2008-2012

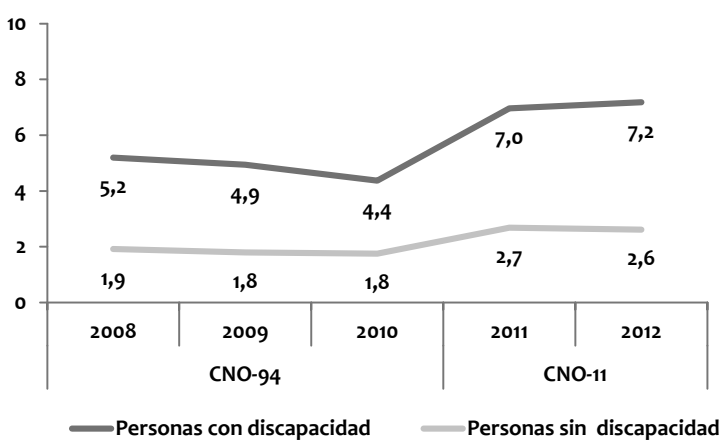

Fuente: INE. El empleo de las personas con discapacidad.

* Nota: Datos 2008-2010 recogidos bajo la clasificación de la CNO-94. Datos 2011-2012 recogidos bajo la clasificación de la CNO-2011.

\section{Salarios más bajos que en la población sin discapacidad, particularmente algunos colectivos}

Según los datos del INE, en 2010 el salario anual bruto de las personas con discapacidad era de 20.553 euros frente a los 22.842,6 euros de las personas sin discapacidad, un $10 \%$ de diferencia. Las desigualdades salariales entre personas con y sin discapacidad se acentúan para algunos colectivos específicos como, por ejemplo, los trabajadores de 30 a 44 años (en donde llegan a ser del 
$21 \%)$, para los trabajadores de la empresa privada (un 17\%) o para perfiles de baja cualificación (un $13 \%$ inferiores).

Si bien es relevante analizar las diferencias intergrupales, esto es, entre personas con y sin discapacidad, son incluso más significativas las desigualdades intragrupo, es decir, dentro del propio colectivo de personas con discapacidad. Atendiendo al tipo de discapacidad, la edad, el nivel educativo, la antigüedad o el grupo de ocupación, las desigualdades salariales existentes son incluso superiores a las que encontramos en las personas sin discapacidad, salvo en el caso del sexo, que la desigualdad entre hombres y mujeres es mayor para personas sin discapacidad.

Las desigualdades son muy evidentes desde el punto de vista del tipo de discapacidad, representando el salario medio de las personas con discapacidad intelectual $(12.710,5 €)$ apenas el $61 \%$ del salario medio del colectivo, mientras que el de las personas con discapacidad sensorial y con discapacidad física es respectivamente un 3,2 y un $4,9 \%$ superior. Atendiendo al grado de discapacidad, observamos que son los de menor grado (33 al 44) y los de mayor (75 y más) los que tienen un salario medio mayor.

Por otro lado, el salario del $10 \%$ de los asalariados con discapacidad que más gana es 4,4 veces superior al salario del $10 \%$ que menos gana.

Si analizamos el salario neto, en lugar del bruto, podemos observar que las diferencias entre personas con y sin discapacidad se reducen. El salario neto mensual de los asalariados con discapacidad es, por término medio, de 1.278,3 euros en 2010, por 1.347 euros de las personas sin discapacidad. La brecha salarial entre trabajadores con discapacidad y sin discapacidad, por lo tanto, se reduce al 5\% una vez descontadas deducciones y cotizaciones a la Seguridad Social, frente al 10\% del salario bruto.

Además de las diferencias integrupales, también se reducen las diferencias salariales intragrupales que mencionábamos antes (por edad, antigüedad, nivel educativo, grupo de ocupación, etc.), una vez descontadas las deducciones y co- tizaciones a la seguridad social. Todos estos datos evidencian que estas medidas indirectas aplicadas sobre el salario en forma de deducciones fiscales o de las cuotas de Seguridad Social, ayudan a reducir las desigualdades existentes.

\section{Desigualdades dentro del colectivo en el acceso al empleo y las condiciones laborales}

Aparte de las diferencias salariales ya mencionadas dentro del colectivo, podemos constatar desigualdades en el acceso al empleo y las condiciones laborales.

A pesar de que la tasa de actividad de la población con discapacidad es baja, observamos diferencias importantes en este indicador en función del sexo, la edad, el nivel de estudios o el tipo y grado de discapacidad. Como ocurre en el caso de las personas sin discapacidad, existen desigualdades de género en el acceso al mercado de trabajo, pues la tasa de actividad es superior en hombres que en mujeres (una diferencia de casi 6 puntos, del 39,2 al 33,5\%).

También podemos observar significativas diferencias por tipo de discapacidad; mientras que las personas con discapacidades sensoriales y físicas tienen una tasa de actividad más alta (57,4\% en auditiva, 42,4\% en visual, 38,8\% física), es muy inferior la participación en el mercado laboral de las personas con discapacidad intelectual $(28,7 \%)$ o enfermedad mental $(27,1 \%)$, lo que puede deberse tanto a las limitaciones en la actividad asociada como en la mayor discriminación existente para estos dos últimos tipos de discapacidad. Las tasas de actividad se reducen conforme mayor es el grado de discapacidad de la persona.

Las diferencias sociodemográficas que mencionábamos en la tasa de actividad se reproducen de manera muy similar en lo que respecta a la tasa de empleo: existen desigualdades de género (significativamente más baja entre las mujeres con discapacidad, un 22,5\%, que entre los hombres, un $26,2 \%$ ), generacionales (muy baja entre los más jóvenes y entre los mayores de 45 años), por 
tipo de discapacidad (dos o tres veces más altas en las sensoriales y físicas que en las intelectuales y mentales) y grado de discapacidad (siendo 3,5 veces mayor entre aquellos con un grado del 33 al $44 \%$ que en aquellos con $75 \%$ y más).

\section{La educación como factor clave (pero no suficiente) para el acceso al mercado laboral}

El acceso a mayores niveles educativos también define sobremanera la relación de las personas con discapacidad con la actividad económica, aumentando la tasa de actividad a medida que aumenta el nivel de estudios máximo alcanzado. Así, sólo un 4,36\% de aquellos sin estudios o analfabetos es activo (una brecha del 91\% con las personas sin discapacidad con el mismo nivel de estudios), por el 57,3\% de aquellos con estudios superiores. La brecha en relación con la población sin discapacidad se reduce a medida que aumenta el nivel de estudios, ya que por lo general un mayor nivel educativo incrementa las oportunidades laborales.

La formación (medida por el nivel de estudios alcanzado) en un factor de mejora de la empleabilidad y reductor de las desigualdades existentes entre personas con y sin discapacidad (lo que podríamos denominar 'brecha de discapacidad'). A mayor nivel de estudios aumenta la tasa de empleo, del 3,1\% en el caso de las personas sin estudios al $45 \%$ entre quienes tienen estudios superiores. La tasa de empleo de los primeros equivale al $12 \%$ de las personas con discapacidad del mismo nivel educativo; en el caso de aquellos con estudios superiores, la tasa de empleo equivale al $61 \%$ de las personas sin discapacidad que tienen el mismo nivel formativo.

\section{Mayor riesgo de pobreza en las personas con discapacidad, aun en los casos en que se encuentran ocupadas}

El riesgo de pobreza entre las personas con discapacidad es mayor, pues tanto ellos como en su hogar experimentan una menor intensidad laboral que en los hogares de personas sin discapacidad (un 31,4\% de "hogares con discapacidad" experimentan baja intensidad laboral por $12,9 \%$ de "hogares sin discapacidad"), situación que prácticamente se ha duplicado con la crisis.

\section{Gráfico 8}

Tasa de baja intensidad laboral de los hogares según tengan personas con discapacidad. Evolución 2004-2012

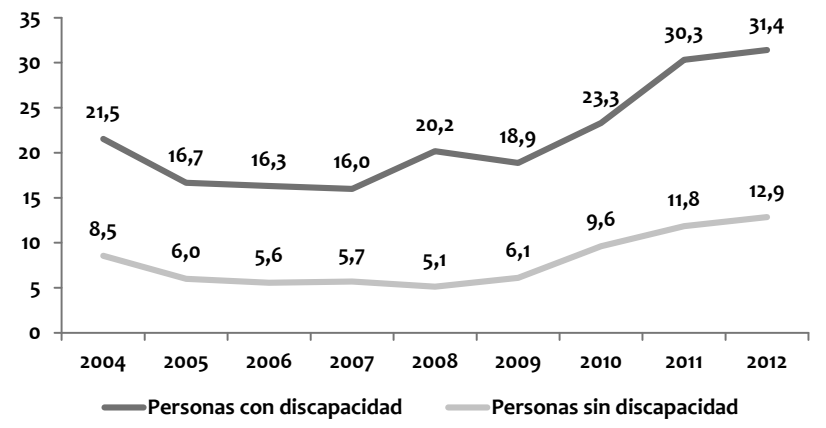

Fuente: INE. Encuesta de Condiciones de Vida.

Aún así, como decíamos, el empleo tampoco es factor suficiente para salir de la pobreza, pues casi un $16 \%$ de los trabajadores con discapacidad se encuentran en riesgo de pobreza, por un $12 \%$ de los trabajadores sin discapacidad.

\section{Gráfico 9}

Evolución de la tasa de riesgo de pobreza de la población con y sin discapacidad ocupada. 2004-2012.

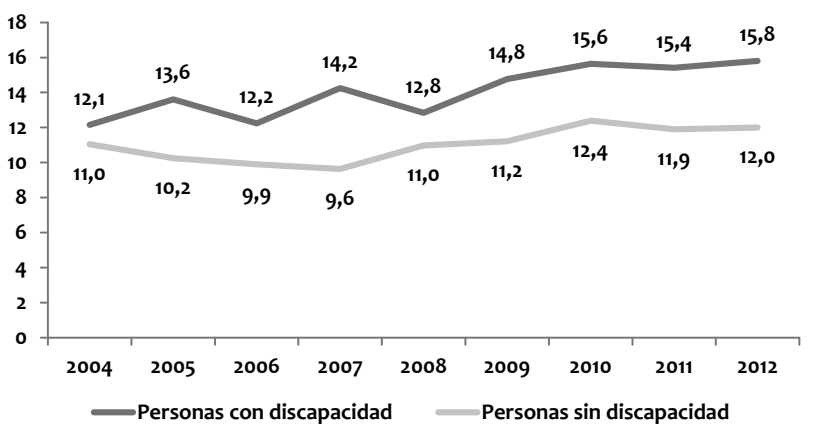

Fuente: INE. Encuesta de Condiciones de Vida. 


\section{La mayoría de la población con discapacidad en edad activa experimenta barreras para acceder al trabajo}

Más del 90\% de la población con discapacidad en edad activa señala una o más barreras para acceder a un empleo adecuado, frente a un $66 \%$ de personas sin discapacidad que lo señalan, según datos de la Encuesta de Integración Social y Salud (EISS) de 2012. Entre las principales barreras que experimenta el colectivo se encuentran, en un $56,7 \%$ de los casos, los motivos de salud o enfermedad, en un $54 \%$ la falta de oportunidades, en un $32,6 \%$ la falta de cualificación o de experiencia y en un $29,1 \%$ las limitaciones en las actividades básicas. La condición de discapacidad, por lo tanto, aumenta las barreras para el acceso a un empleo adecuado.

\section{Gráfico 10}

Población con discapacidad y sin discapacidad en edad activa en función de la existencia de barreras para el acceso a un empleo adecuado. 2012

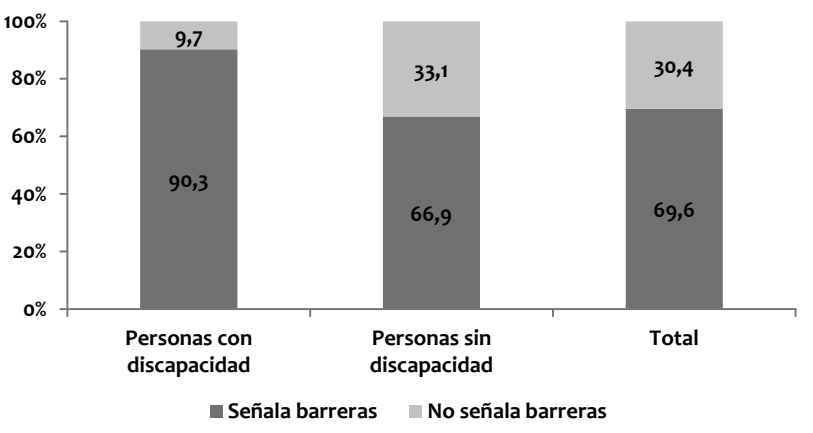

Fuente: INE. Encuesta de Integración Social y Salud (EISS 2012).

Como podemos ver, las barreras para el acceso al mercado laboral afectan prácticamente a toda la población con discapacidad en edad activa. Pero, ¿cómo es la vivencia de esas personas que acceden al mercado de trabajo, en concreto, de los jóvenes con discapacidad que acceden al mercado laboral en el contexto de crisis? Antes decíamos que el mayor nivel educativo favorecía la inserción, pero que no era condición suficien- te, especialmente para aquellas personas con discapacidad más jóvenes que sufren altas tasas de paro.

Si bien muchas personas con discapacidad han experimentado ya barreras o segregación en el ámbito educativo, con altas tasas de abandono escolar precoz (de un 43,2\% entre jóvenes de 18 a 24 años), la finalización del periodo educativo y los primeros acercamientos al mundo laboral suelen suponer una ruptura con la trayectoria vital, pues el esfuerzo y la superación, que para algunos ha servido para alcanzar los niveles superiores en el sistema educativo, ya no son condición suficiente para alcanzar la meta del acceso a un empleo cualificado, sino que los factores externos cobran gran importancia. El discurso de los jóvenes con discapacidad es el de la frustración y el desengaño: "He hecho toda la vida lo que se supone que había que hacer, ¿no? ¿Por qué no consigo un empleo?".

Tras un camino de esfuerzo continuo para alcanzar una elevada cualificación, la persona se encuentra sin empleos para su perfil o con becas eventuales escasamente remuneradas para hacer algo lejano a aquello para lo que estuvo años formándose. Algunos entrevistados prefieren ocultar en los procesos de selección su discapacidad, salvo en el mismo momento de la entrevista, pues aunque ellos lo ven como una muestra de superación, consideran que la empresa lo ve como un inconveniente, por desconocimiento y/o por desinterés.

"Bueno, no sé muy bien qué hacer con esto de las entrevistas de... de esto de si decir o no, que soy discapacitado. Nunca sabes si es beneficioso o perjudicial, ¿no? Si lo digo, para la empresa va a tener beneficios fiscales y tal, pero si lo digo a lo mejor el de la empresa se cree que no me voy a saber manejar y tal y dice - "Uy, uy, no, no yo no quiero tener problemas"- y no me contra$t a$, sin verme en persona, sin verme que yo me manejo, pues... claro pues a lo mejor no quiero andar diciéndolo." 
Es habitual entre aquellos perfiles cualificados considerar que las bolsas de empleo y recursos de entidades de discapacidad intermediadoras en el acceso al empleo no están suficientemente orientadas hacia sus perfiles por lo que, aunque se apuntan a ellas, sus expectativas con las mismas son escasas, pues como veíamos, los trabajos "reservados" para el colectivo están segmentados a actividades sectorizadas y de baja cualificación:

"En Fundación Adecco (...) y en la bolsa de empleo de la ONCE estoy apuntado... Entonces qué pasa, que estos fondos de empleo pues supongo que las empresas más o menos conocen el tipo de demanda, el tipo de gente con discapacidad que suele venir, el perfil de gente que va, de gente no tan cualificada"

\section{Las políticas de empleo se dirigen principalmente al mantenimiento de puestos de trabajo}

El gasto en políticas de empleo con apoyo y rehabilitación en España es, para el año 2011 (último dato disponible), de 851,102 millones de euros. Esta cantidad supone un 2,2\% sobre el total del gasto en políticas de empleo y un $11,5 \%$ del gasto en políticas activas de empleo en nuestro país para el año en cuestión, por un $0,08 \%$ sobre el total del PIB.

Este gasto ha crecido en los últimos años. Especialmente en 2007 estas medidas experimentaron un crecimiento significativo (pasando de 208 a 640 millones y de un 1 a un 2,8\% del PIB), probablemente impulsadas por los avances legislativos producidos por esas fechas. Desde ese año han experimentado un crecimiento sostenido, salvo para el año 2011, donde se redujo en 9 millones con respecto a 2010.

Sin embargo, el gasto en ayudas concedidas a la integración laboral de personas con discapacidad para 2012 fue de $259.340 .600 €$, una reducción de un $20 \%$ con respecto al año anterior. Este gasto supone un $74,3 \%$ del total del gasto en ayudas de apoyo a la creación de empleo y un $0,025 \%$ del
PIB. De estas ayudas, la mayoría (el 99,7\%) van a Centros Especiales de Empleo, efectuándose gran parte del gasto para el mantenimiento de puestos de trabajo $(91,2 \%)$ y en menor medida para proyectos generadores de empleo $(2,1 \%)$ y Unidades de Apoyo (6,4\%). El restante $0,3 \%$ va destinado a proyectos de Empleo con Apoyo. Como decíamos, para 2012 se ha experimentado una reducción importante del gasto, que también hubo para 2011. Para el año 2012, el número de beneficiarios de ayudas concedidas a la integración laboral de personas con discapacidad es de 81.560, siendo en su mayoría para el mantenimiento del puesto de trabajo (61.851), seguidos de los beneficiarios de unidades de apoyo (19.142).

Vemos, por lo tanto, que los recursos económicos se centran en el empleo protegido. Atendiendo a datos de la serie 'El empleo de las personas con discapacidad' se puede inferir que las empresas incumplen sistemáticamente la cuota de reserva del $2 \%$ (sólo el $18,6 \%$ de trabajadores de empresas de 50 o más trabajadores están empleados en empresas que cumplen dicha cuota).

La misma fuente nos muestra que sólo uno de cada cuatro trabajadores con discapacidad cotiza con bonificaciones o reducciones en las cuotas de cotización, la misma proporción de trabajadores con discapacidad que cuenta con contratos específicos de discapacidad.

\section{Gráfico 11}

Asalariados con contrato específico de discapacidad 2008-2012

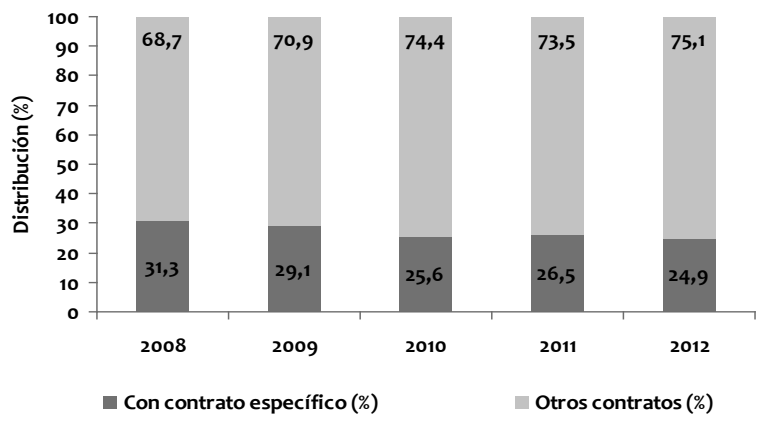

Fuente: INE, El empleo de las personas con discapacidad. 


\section{DISCUSIÓN}

Como planteábamos y hemos podido comprobar con algunos datos y reflexiones, la población con discapacidad tiene muchas dificultades para el acceso al empleo, existiendo desigualdades importantes entre el colectivo y la población sin discapacidad. En ese sentido, la normativa nacional e internacional dirigida al colectivo es papel mojado, por la clara incompatibilidad entre las condiciones y exigencias productivas del mercado de trabajo en el contexto del nuevo capitalismo feudal y los derechos humanos o de ciudadanía (social, civil, político). Estos derechos, formalmente plasmados en la Convención o en otras normas, no se hacen efectivos en el actual contexto económico, social y político, con unas políticas sociales públicas en regresión y cada vez más orientadas a la individualización de las problemáticas sociales, la precarización y el debilitamiento de los derechos sociales y laborales.

- Esta dinámica, que es común a todos los trabajadores en el contexto neoliberal actual de internacionalización de la precariedad, se agudiza particularmente en el caso de colectivos como el de las personas con discapacidad, debido a las condiciones de desigualdad y a las particulares barreras, estructurales y simbólicas, que impiden al colectivo el acceso a los espacios de ciudadanía en las mismas condiciones que el resto de la población. Y la crisis agudiza esas desigualdades.

- En este trabajo nos preguntábamos por dichas desigualdades. En concreto, veamos algunas conclusiones relevantes a partir de los datos analizados:

- Con la crisis ha aumentado la tasa de actividad al tiempo que se ha observado una acentuada caída del empleo y un crecimiento notable de la tasa de paro, incrementándose además el paro de larga duración y el índice de rotación en la contratación.
- Si bien las personas con discapacidad tienen, como bien es sabido, una peor posición en el mercado de trabajo que las personas sin discapacidad, la desigualdad también existe dentro del propio colectivo, incluso acentuándose la dualización para algunas variables. Esto es especialmente representativo en lo que respecta a las tasas de temporalidad, a los salarios o a la contratación a tiempo parcial, donde es evidente la desigual posición de las mujeres con discapacidad frente a los hombres (aunque menor que en el colectivo de personas sin discapacidad). También es evidente la desigualdad por tipo de discapacidad, así como la mayor estratificación salarial entre el colectivo atendiendo a variables como el nivel educativo o el grupo de ocupación.

- Si analizamos la estructura ocupacional, podemos comprobar que la mayoría de los asalariados con discapacidad tienen contrato indefinido, aunque 9 de cada 10 contratos nuevos son temporales El trabajo a tiempo parcial es mucho más habitual en mujeres que en hombres con discapacidad. La mayoría de los trabajadores con discapacidad se emplean en ocupaciones de cualificación media y baja. Sin embargo, la nueva contratación de las personas con discapacidad se caracteriza por una creciente inestabilidad y precariedad, sectorizada en pocas actividades económicas y segmentada en ocupaciones elementales. La tendencia es que las condiciones laborales de la nueva contratación de personas con discapacidad sean cada vez más precarias (mayor temporalidad, mayor contratación a tiempo parcial, etc.), lo que acentúa la dualización del colectivo dentro del mercado laboral, entre los nuevos que acceden a él y los que entraron en el pasado con una buena posición en términos de cualificación, antigüedad, etc.

- Casi uno de cada seis trabajadores con discapacidad está en riesgo de pobreza a pesar de encontrarse ocupados, el 16\% de las personas con discapacidad ocupadas se podían conside- 
rar trabajadores pobres para el año 2012. Esa tasa de las personas con discapacidad multiplica a la del resto de la población por 1,31 (es decir, es un $31 \%$ más elevada), lo que implica que el tener un empleo no es condición suficiente para que una persona con discapacidad salga de la pobreza. La actividad laboral de las personas con discapacidad es menor, pero también es menor la intensidad laboral de los hogares en los que habitan, probablemente por la implicación de las necesidades de apoyos y cuidados: 1 de cada 3 personas con discapacidad viven en hogares con baja intensidad laboral.

- Con respecto a las políticas de empleo dirigidas a las personas con discapacidad, los datos muestran que estas políticas tienen un mayor impacto en el mantenimiento del puesto de trabajo que en la creación de nuevo empleo (progresivamente más inestable), lo que no contribuye a disminuir la dualización laboral. El gasto en políticas de empleo con apoyo y rehabilitación fue aumentado hasta 2011, beneficiándose más de 70.000 personas se benefician de las políticas de empleo con apoyo y rehabilitación. En 2012, sin embargo, descendió un $20 \%$ el gasto en ayudas a la integración laboral del colectivo, siendo esas ayudas destinadas en su mayoría al mantenimiento de los puestos de trabajo en centros especiales de empleo. El gasto medio por beneficiario de las ayudas concedidas a la integración laboral de personas con discapacidad es de 3.166,6 euros.

- La cobertura de las medidas de acción positiva de promoción del empleo del colectivo es bastante bajo, aun demostrando su impacto para reducir desigualdades con respecto a la población sin discapacidad. Sólo uno de cada cuatro trabajadores con discapacidad cotiza en 2012 con bonificaciones o reducciones en las cuotas de cotización. También uno de cada cuatro tiene contrato específico de discapacidad, más común en asalariados con discapaci- dad intelectual, si bien ha quedado demostrado que estas medidas reducen las diferencias salariales. Además, se puede afirmar que la mayoría de las empresas de más de 50 trabajadores incumplen la cuota de reserva del $2 \%$. Por lo tanto, las medidas que buscan un impacto en la estructura apenas tienen alcance (cuotas de reserva) y las que dan respuestas individuales (reducciones, bonificaciones), mejoran las condiciones laborales pero apenas cubren a un pequeño sector del colectivo de personas con discapacidad.

\section{REFERENCIAS BIBLIOGRÁFICAS}

1. España. Real Decreto Legislativo 1/2013, de 29 de noviembre, por el que se aprueba el Texto Refundido de la Ley General de derechos de las personas con discapacidad y de su inclusión social, Boletín Oficial del Estado, núm. 289 de 3 de diciembre de 2013, p. 95635-95673. (en línea) https://www.boe.es/boe/ dias/2013/12/03/pdfs/BOE-A-2013-12632.pdf〉, acceso marzo de 2014.

2. Laloma, M. (2007). Empleo protegido en España. Análisis de la normativa legal y logros alcanzados. Madrid: CERMI.

3. Morris, J. (1998). Citizenship, self-determination and political action: the forging of a political movement. Conference on Citizenship and Disability. Sydney, Australia.

4. Morris, J. (2005). Citizenship and disabled people: A scoping paper prepared for the Disability Rights Commission. Leeds: Disability Archive UK.

5. Marks, D. (2001). Disability and cultural citizenship: exclusion, integration and resistance, en Stevenson, Nick (ed.): Culture \& Citizenship. London: Sage.

6. Young, I. M. (2000). La justicia y la política de la diferencia. Madrid: Editorial Cátedra.

7. Díaz Velázquez, E. (2010). Ciudadanía, identidad y exclusión social de las personas con discapacidad. Política y Sociedad, V47. N1., 131-145.

8. Marshall, T.H.; Bottomore, T. (1998). Ciudadanía y Clase Social. Madrid: Alianza Editorial.

9. Oliver, M. (2008). Políticas sociales y discapacidad. Algunas consideraciones teóricas. En Barton, L. (comp.): Superar las barreras de la discapacidad. Madrid: Ediciones Morata, 19-33.

10. Alonso, L. E. (2000). Ciudadanía, sociedad del trabajo y Estado de Bienestar: los derechos sociales de la era de la fragmentación. En M. Pérez Ledesma (ed.): Ciudadanía y democracia, Madrid: Editorial Pablo Iglesias. 159-189.

11. Abberley, P. (1998). Trabajo, utopía e insuficiencia. En Len Barton (comp.): Discapacidad y sociedad. Madrid: Ediciones Morata. 77-96. 
12. Castells, M. (2001): La era de la información. Volumen III. México: Editorial Siglo XXI.

13. Tezanos (2001). El trabajo perdido. ¿Hacia una civilización postlaboral? Madrid: Biblioteca Nueva.

14. Organización Mundial de la Salud, OMS (2001). Clasificación Internacional de Funcionamiento, de la Discapacidad y de la Salud. Madrid: Instituto de Migraciones y Servicios Sociales (IMSERSO).

15. Instituto Nacional de Estadística (2012). El Empleo de las personas con discapacidad. (en línea) http://www.ine.es/jaxi/menu. do? type $=$ pcaxis $\&$ path $=/$ t22 $/$ p320\&file $=$ inebase $\& L=0\rangle, \quad$ acceso marzo de 2014.

16. Instituto Nacional de Estadística (2010): El salario de las personas con discapacidad. (en línea) http://www.ine.es/jaxi/menu. do? type $=$ pcaxis $\&$ path $=/ \mathrm{t} 22 / \mathrm{p} 331 \&$ file $=$ inebase $\& \mathrm{~L}=0$; , acceso marzo de 2014.

17. Servicio Público de Empleo Estatal (2014). Datos estadísticos de contratos. (en línea) 〈http://www.sepe.es/contenido/estadisticas/ datos_estadisticos/contratos/datos/estadisticas_nuevas.html?, acceso marzo de 2014.
18. Observatorio de las Ocupaciones (2013). Informe del Mercado de Trabajo de las personas con discapacidad 2012. Servicio Público de Empleo Estatal (SEPE).

19. Instituto Nacional de Estadística (2012). Encuesta de Integración Social y Salud. (en línea) ‘http://www.ine.es/jaxi/menu.do?type= pcaxis\&path=\%2Ft15\%2Fp470\&file=inebase , acceso marzo de 2014.

20. Eurostat (2013). Income and living conditions, EU-SILC. (en línea) «http://epp.eurostat.ec.europa.eu/portal/page/portal/income_social_inclusion_living_conditions/data/database», acceso marzo de 2014.

21. Zaidi, A. (2011): The situation of working-age people with disabilities across the EU. Research Note 5 /2011. European Commission, Social Europe. (en línea) http://www.euro.centre.org/ data/1364397289 92141.pdf>, acceso 21 de marzo de 2014.

22. Eurostat (2013). The labour market policy database. (en línea) «http://epp.eurostat.ec.europa.eu/portal/page/portal/labour_market/labour_market_policy/database», acceso marzo de 2014.

23. Ministerio de Empleo y Seguridad Social (2013). Anuario de estadísticas del Ministerio de Empleo y Seguridad Social. (en línea) 〈http://www.empleo.gob.es/es/estadisticas/contenidos/anuario. htm), acceso marzo de 2014. 